\title{
SITUAÇÕES DE VULNERABILIDADE EM SAÚDE DE PESSOAS COM DOENÇAS CARDIOVASCULARES: SÍNTESE DO CONHECIMENTO
}

\section{SITUATIONS OF VULNERABILITY IN THE HEALTH OF PEOPLE WITH CARDIOVASCULAR DISEASES: SYNTHESIS OF KNOWLEDGE}

\author{
Ingrid Kelly Morais Oliveira ${ }^{1}$ Francisco Douglas Canafístula de Souza $^{2} *$ Tatiane de Sousa Paiva $^{3}$ \\ Keila Maria de Azevedo Ponte Marques ${ }^{4}$
}

\begin{abstract}
RESUMO
Objetivo: Descrever a síntese do conhecimento das vulnerabilidades em saúde no adoecimento cardiovascular. Método: Trata-se de uma revisão integrativa elaborada de agosto a novembro de 2021 a partir da questão norteadora: Quais as situações de vulnerabilidade em saúde de pessoas com Doenças Cardiovasculares? Encontrou-se um total de 41 artigos científicos, com aplicação dos critérios de inclusão e exclusão obteve-se uma amostra final de nove artigos. Resultados: Dentre os aspectos relacionados a vulnerabilidade em saúde no adoecimento cardiovascular destacam-se: tabagismo, baixa escolaridade, consumo de bebidas alcoólicas, má alimentação, sedentarismo, obesidade, hipertensão arterial, diabetes mellitus, triglicerídeos altos, falta de acesso à informação, exposição a alta temperatura e poluição do ar, histórico de internações hospitalares, depressão, sintomas psiquiátricos e qualidade do sono. Considerações Finais: As situações vulnerabilizantes no adoecimento cardiovascular evidenciadas no estudo estão prioritariamente relacionados aos fatores de risco cardiovascular, torna-se inescusável a produção científica voltada a outras questões que afetam essa condição humana.

Palavras-chave: Vulnerabilidade em Saúde; Doenças Cardiovasculares; Fatores de Risco de Doenças Cardíacas; Enfermagem Cardiovascular; Literatura de Revisão como Assunto.
\end{abstract}

\begin{abstract}
Objective: To describe the synthesis of knowledge on health vulnerabilities in cardiovascular disease. Method: This is an integrative review elaborated from August to November 2021 from the guiding question: What are the health vulnerability situations of people with Cardiovascular Diseases? A total of 41 scientific articles were found, with application of the inclusion and exclusion criteria, a final sample of nine articles was obtained. Results: Among the aspects related to health vulnerability in cardiovascular disease, the following stand out: smoking, low education, alcohol consumption, poor diet, sedentary lifestyle, obesity, hypertension, diabetes mellitus, high triglycerides, lack of access to information, exposure high temperature and air pollution, history of hospital admissions, depression, psychiatric symptoms and sleep quality. Final Considerations: The vulnerable situations in cardiovascular disease evidenced in the study are primarily related to cardiovascular risk factors, making scientific production focused on other issues that affect this human condition is inexcusable.

Keywords: Health Vulnerability; Cardiovascular Diseases; Risk Factors for Heart Disease; Cardiovascular Nursing; Review Literature as Topic.
\end{abstract}

\footnotetext{
${ }^{1}$ Universidade Estadual Vale do Acaraú, Sobral, Ceará, Brasil. https://orcid.org/0000-0003-1536-7289.

${ }^{2}$ Universidade Estadual Vale do Acaraú, Sobral, Ceará, Brasil. https://orcid.org/0000-0002-8845-1062.

${ }^{3}$ Universidade Estadual Vale do Acaraú, Sobral, Ceará, Brasil. https://orcid.org/0000-0001-8555-6355.

${ }^{4}$ Universidade Estadual do Ceará, Sobral, Ceará, Brasil. https://orcid.org/0000-0001-5215-7745.
} 


\section{INTRODUÇÃO}

A Vulnerabilidade em Saúde (VS), uma condição humana fruto da interação entre sujeito e social ao exprimir valores multidimensionais, tem como potencialidade a interpretação e intervenção no processo saúde doença. Por isso a necessidade da identificação dos aspectos vulnerabilizantes de pessoas com Doenças Cardiovasculares (DCV), pois representam a principal causa de mortes no Brasil desde a década de 60, com mais de 1100 mortes por dia ${ }^{(1)}$.

Trata-se de um conceito ainda recente no meio científico e epidemiológico, no entanto, o seu desenvolvimento foi influenciado durante o Movimento da Reforma Sanitária Brasileira (1970) na forma como a população reagiu ao dramático quadro do setor da saúde ${ }^{(2)}$. Vem sendo utilizado em diferentes regiões do mundo, com destaque para a América do Sul, especificamente no Brasil, isto se deve a questão histórica. Tratase de uma ideia importante para a saúde coletiva em todo o mundo ${ }^{(3)}$.

À vista do exposto, define-se vulnerabilidade em saúde como uma condição humana desenvolvida a partir da interação entre o sujeito e o meio social em que convive, de modo que há uma relação de poder que se movimenta em direção a uma condição precária quando não há emancipação por parte do sujeito ou do coletivo para mudar esta situação(4). Assim este conceito configura-se pelo fato de o https://doi.org/10.31011/reaid-2021-v.95-n.36-art.1267 Rev Enferm Atual In Derme v. 95, n. 36, 2021 e-021178 sujeito estar envolvido por um conjunto de atributos que o condicionam a esta situação, desta forma diz-se que as pessoas estão vulneráveis a algo ou alguém em determinado ponto do tempo ou espaço, e não que elas são vulneráveis ${ }^{(5)}$.

Assim, umas das potencialidades do conceito de Vulnerabilidade em Saúde é a possibilidade de interpretar a doença e propor intervenções capazes de modificar a situação de saúde-doença, com vistas a superar a abordagem biológica e comportamental, identificando e intervindo na dimensão individual e coletiva ${ }^{(6)}$.

Deste modo, como as DCVs levam a complicações, em sua maioria, degenerativas provocando inúmeras alterações prejudiciais para o corpo humano, considera-se vulnerável para complicações cardiovasculares, qualquer pessoa, que não possua informação suficiente do processo saúde-doença-cuidado, quando não valoriza as alterações físicas em seu próprio corpo, quando não reconhece os sintomas da doença que possui, quando se automedica e não se importa com a sua saúde ${ }^{(7)}$.

Ademais, essas doenças são a principal causa de mortalidade no Brasil desde a década de 60, com um quantitativo de mais de 1100 mortes por dia, causando 2,3 vezes mais mortes que todas as causas externas e 6,5 vezes mais que todos os tipos de infecções incluindo a AIDS, tornando-se 
assim uma grande preocupação para os gestores de saúde que buscam formas de minimizar a ocorrência destes agravos ${ }^{(8)}$.

Todavia, ao considerar o paciente cardiopata como ser vulnerável, não se deve intervir somente nas complicações propriamente dita, mas agir antecipadamente naquilo que irá determiná-las. Desta forma, não se pode pensar ou planejar o cuidado de forma pontual, e sim, por meio de um olhar $\operatorname{ampliado}^{(9)}$.

Com base no exposto, esse estudo tem impacto no desenvolvimento científico por abordar um assunto emergente ao identificar e apresentar as situações de vulnerabilidade em saúde de pessoas com DCV e os resultados dessa pesquisa irão contribuir no desenvolvimento social no que se refere a clarificar as situações de vulnerabilidade em saúde de pessoas cardiopatas

Este estudo teve como objetivo descrever a síntese do conhecimento das vulnerabilidades em saúde no adoecimento cardiovascular.

\section{MÉTODOS}

Trata-se de uma revisão integrativa da literatura, a qual permite realizar a busca, avaliação crítica e a síntese de resultados de pesquisas sobre a temática pesquisada, desta forma contribuindo para a implementação de intervenções efetivas na assistência à saúde e avanço do conhecimento ${ }^{(10)}$. Nesse estudo foram adotadas as etapas propostas por
Mendes, Silveira e Galvão (2019) ${ }^{(11)}$ :

Identificação do tema ou formulação da questão norteadora; (2) Estabelecimento de critérios para inclusão e exclusão dos estudos; (3) Definição das informações a serem extraídas dos estudos selecionados; (4) Avaliação dos estudos incluídos na revisão integrativa; (5) Interpretação dos resultados e (6) Síntese do Conhecimento.

Assim, na primeira etapa foi delineada a questão norteadora: Quais as situações de vulnerabilidade em saúde de pessoas com DCV? Para a etapa seguinte, realizada em agosto de 2021, ocorreu o estabelecimento dos critérios de inclusão e exclusão. A busca dos artigos ocorreu por meio dos descritores “doenças cardiovasculares OR cardiovascular diseases" e "vulnerabilidade em saúde OR health vulnerability", mediados pelo operador booleano "AND.", estes descritores foram extraídos do DECS (Descritores em Ciências da Saúde) do Portal BVS (Biblioteca Virtual em Saúde). Para a seleção dos artigos, utilizaram-se as bases de dados: PUBMED Central: PMC e SCOPUS, e nas Bibliotecas Virtuais: SCIELO e Biblioteca Virtual em Saúde (BVS). Outrossim, empregou-se da estratégia PICO, que representa um acrônimo para Paciente, Intervenção, Comparação e “Outcomes" (desfecho). A utilização desta estratégia auxilia nas definições da pesquisa, visto que, orienta a construção da pergunta de pesquisa e da busca bibliográfica permitindo ao pesquisador localizar de forma rápida a 
melhor informação científica ${ }^{(12)}$. Para este estudo utilizou-se: P (Pessoas com DCV); I (); C (Pessoas sem DCV); O (Vulnerabilidades em Saúde).

Encontrou-se um total de 46 artigos científicos nas quatro bases mencionadas, vale salientar, que não foram utilizadas outras bases por não haver estudos sobre a temática. Os critérios de inclusão estabelecidos para a revisão foram artigos de pesquisa: que respondessem à questão norteadora do estudo, disponíveis eletronicamente na íntegra nos idiomas inglês, português ou espanhol sem delimitação de tempo. Foram excluídos: publicações repetidas, estudos de revisão, cartas ao editor, editoriais e que não se relacionassem com a temática. Logo, a amostra final foi composta por nove artigos que atendiam aos critérios de inclusão e exclusão estabelecidos, conforme fluxograma PRISMA (Figura 1).

Figura 1- Fluxograma PRISMA de identificação do processo de seleção dos estudos para compor a revisão integrativa. Sobral - CE, Brasil, 2021

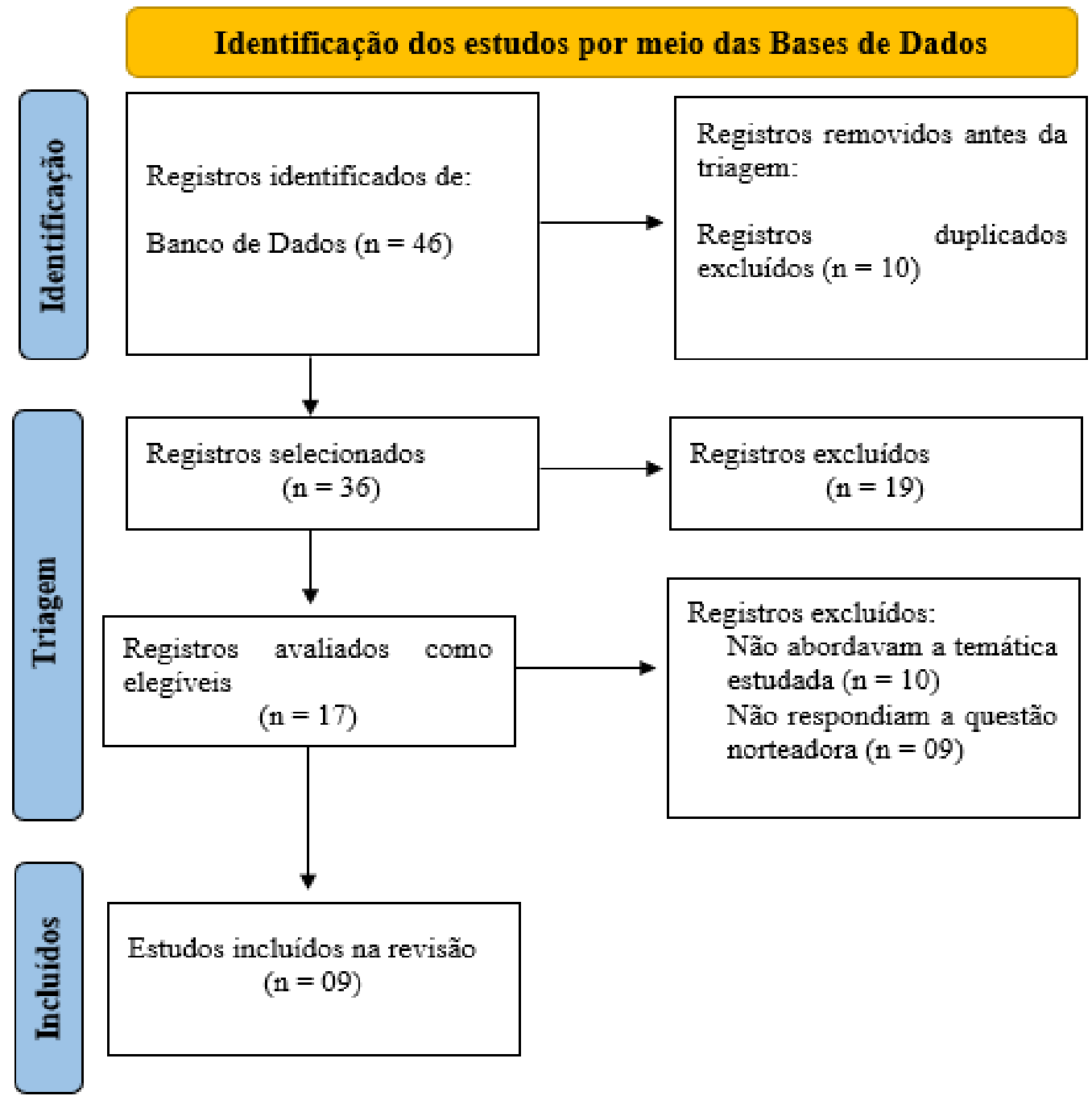

Fonte: Os autores 
De acordo com a $3^{\mathrm{a}}$ etapa ocorreu a definição das informações a serem extraídas dos estudos selecionados, para isto utilizou-se o formulário adaptado ${ }^{(13)}$, o qual foi preenchido para cada artigo da amostra final, contemplando as seguintes informações: Base de dados, identificação do artigo (título do artigo, país, idioma, ano de publicação e nível de evidência), objetivo, método e situações de VS nas DCV.

Nas etapas seguintes, ocorreu a avaliação dos estudos incluídos na revisão integrativa com interpretação dos resultados $^{(14)}$. Assim, os dados foram analisados de forma detalhada e crítica, buscando identificar as situações de vulnerabilidade em saúde no adoecimento cardiovascular, refletindo e buscando explicações para os resultados diferentes e procedendo a complementaridade.

Para a última etapa da Revisão Integrativa, a síntese do conhecimento foi apresentada por meio da construção de um quadro com as principais informações obtidas no estudo.

\section{RESULTADOS}

Ao final foram totalizados nove artigos, a partir destes propiciou-se a criação do banco de dados, organizados em um quadro analítico (Quadro 1) contido das seguintes variáveis: título, país, idioma, ano de publicação e nível de evidência proposto por Melnyk e Fineout-Overholt, 2011 $1^{(15)}$ (objetivo, método e situações de VS nas DCV).

Os artigos, foram selecionados de acordo com os critérios de inclusão previamente estabelecidos, sendo encontrados nas bases de dados PUBMED cinco artigos, SCIELO cerca de dois, somente na SCOPUS um e BVS apenas um. Pode-se visualizar que não há uma base de dados que publique de maneira substancial pesquisas sobre as vulnerabilidades geradoras do adoecimento cardiovascular. A partir da análise dos países de publicação, observou-se que havia mais publicações internacionais, cerca de sete artigos internacionais, e apenas dois de periódicos nacionais. Assim, pode-se afirmar que a comunidade científica brasileira precisa ser fortalecida na pesquisa (Quadro 1).

Quadro 1 - Caracterização das publicações incluídas na revisão, de acordo com a base de dados, título, país e periódico. Sobral - CE, Brasil, 2021

\begin{tabular}{|c|c|c|c|c|}
\hline $\mathbf{N}^{\mathbf{0}}$ & Base de Dados & Título do Periódico & País & Periódico \\
\hline 1 & PUBMED & $\begin{array}{l}\text { Prevalência De Fatores De Risco Para Doenças Cardiovasculares: } \\
\text { Um Estudo Transversal Baseado Na Comunidade Em Uma } \\
\text { Comunidade Periurbana De Kathmandu, Nepal }\end{array}$ & Índia & $\begin{array}{ll}\text { Indian } & \text { Heart } \\
\text { Journal } & \end{array}$ \\
\hline 2 & PUBMED & $\begin{array}{l}\text { Tendências Temporais Da Associação Entre Temperatura } \\
\text { Ambiente E Hospitalizações Por Doenças Cardiovasculares Em } \\
\text { Queensland, Austrália De 1995 A 2016: Um Estudo De Caso- } \\
\text { Cruzado Estratificado No Tempo }\end{array}$ & Austrália & $\begin{array}{l}\text { PLOS } \\
\text { Medicine }\end{array}$ \\
\hline
\end{tabular}




\begin{tabular}{|c|c|c|c|c|}
\hline 3 & PUBMED & $\begin{array}{l}\text { Associação Entre Poluição Ambiente Por Partículas Finas E } \\
\text { Internações Hospitalares Por Causa De Doença Cardiovascular } \\
\text { Específica: Estudo De Série Temporal Em } 184 \text { Grandes Cidades } \\
\text { Chinesas }\end{array}$ & China & The BMG \\
\hline 4 & PUBMED & $\begin{array}{l}\text { Trajetórias De Emprego Ao Longo Da Vida: Uso De Tabaco E } \\
\text { Consumo De Álcool E Doenças Cardiovasculares Na Velhice }\end{array}$ & Chile & $\begin{array}{l}\text { SSM - } \\
\text { Population He } \\
\text { alth }\end{array}$ \\
\hline 5 & PUBMED & $\begin{array}{l}\text { Mudanças Na Vulnerabilidade Entre Pacientes Idosos Com Doença } \\
\text { Cardiovascular Nos Primeiros } 90 \text { Dias Após A Alta Hospitalar: } \\
\text { Uma Análise Secundária De Um Estudo De Coorte }\end{array}$ & EUA & BMJ Open \\
\hline 6 & SCOPUS & $\begin{array}{l}\text { Risco Cardiovascular Como Moderador De Associações Entre } \\
\text { Sensibilidade À Ansiedade, Tolerância Ao Estresse, PTSD E } \\
\text { Sintomas De Depressão Entre Bombeiros Expostos A Traumas }\end{array}$ & EUA & $\begin{array}{l}\text { Journal of } \\
\text { Psychosomatic } \\
\text { Research }\end{array}$ \\
\hline 7 & SCIELO & $\begin{array}{l}\text { Associação entre condições de vida e vulnerabilidade com a } \\
\text { mortalidade por doenças cardiovasculares de homens idosos do } \\
\text { Nordeste }\end{array}$ & Brasil & $\begin{array}{l}\text { Revista } \\
\text { Brasileira de } \\
\text { Epidemiologia }\end{array}$ \\
\hline 8 & SCIELO & $\begin{array}{l}\text { Variabilidade da temperatura do ar diária, vulnerabilidade social e a } \\
\text { relação com altas taxas de mortalidade por doenças } \\
\text { cardiovasculares na Colômbia }\end{array}$ & Colômbia & USP \\
\hline 9 & BVS & $\begin{array}{l}\text { Fatores De Risco Cardiovasculares E Qualidade De Vida Em } \\
\text { Universitários }\end{array}$ & Brasil & $\begin{array}{l}\text { Revista de } \\
\text { Enfermagem } \\
\text { UFPE on line }\end{array}$ \\
\hline
\end{tabular}

Fonte: Elaborado pelos autores (2021).

Ao realizar comparação entre os objetivos dos estudos analisados, respectivamente os nove artigos, possuíam a mesma temática da questão norteadora do estudo. A partir dessa compatibilidade de assunto, pode-se averiguar quão interessante e importante é o assunto para fomento do conhecimento científico e assim proporcionar melhores trajetos na promoção à saúde cardiovascular (Quadro 2).

Quadro 2 - Caracterização das publicações incluídas na revisão, de acordo com o número, objetivo, tipo de estudo e nível de evidência. Sobral - CE, Brasil, 2021

\begin{tabular}{|c|c|c|c|}
\hline $\mathbf{N}^{\mathbf{0}}$ & Objetivo & Tipo de Estudo & Nível de Evidência \\
\hline 1 & $\begin{array}{l}\text { Identificar a prevalência e a distribuição sociodemográfica dos } \\
\text { fatores de risco para doenças cardiovasculares }\end{array}$ & $\begin{array}{l}\text { Estudo } \\
\text { transversal }\end{array}$ & III \\
\hline 2 & $\begin{array}{l}\text { Investigar as variações temporais na associação entre temperatura } \\
\text { ambiente e hospitalizações por doenças cardiovasculares }\end{array}$ & Meta-analise & $\mathrm{I}$ \\
\hline 3 & $\begin{array}{l}\text { Estimar os riscos de internações hospitalares diárias por causa de } \\
\text { doenças cardiovasculares principais específicas associadas à } \\
\text { exposição de curto prazo à poluição por partículas finas do ambiente } \\
\text { (diâmetro aerodinâmico } \leq 2,5 \mu \mathrm{m} ; \mathrm{PM} \text { ) na China. }\end{array}$ & Meta-analise & $\mathrm{I}$ \\
\hline 4 & $\begin{array}{l}\text { Reconstruir o emprego simultâneo, o uso do tabaco, e trajetórias de } \\
\text { consumo de álcool ao longo da vida (do nascimento à velhice) e para } \\
\text { medir a associação entre essas trajetórias e DCV na velhice. }\end{array}$ & $\begin{array}{l}\text { Estudo de } \\
\text { coorte }\end{array}$ & IV \\
\hline 5 & $\begin{array}{l}\text { Comparar as mudanças na vulnerabilidade após a alta hospitalar } \\
\text { entre pacientes mais velhos com DCVs que receberam alta para casa } \\
\text { com autocuidado versus encaminhamento de saúde domiciliar. }\end{array}$ & Meta-análise & $\mathrm{I}$ \\
\hline 6 & $\begin{array}{l}\text { Investigar o risco cardiovascular como moderador de associações } \\
\text { entre fatores de risco (AS e DT) e sintomas psiquiátricos (PTSD e } \\
\text { depressão) entre bombeiros. }\end{array}$ & Ensaio Clínico & III \\
\hline 7 & $\begin{array}{l}\text { Identificar fatores explicativos da taxa de mortalidade por DCVs dos } \\
\text { homens idosos no Brasil, a partir de indicadores das condições de }\end{array}$ & $\begin{array}{l}\text { Estudo } \\
\text { ecológico com }\end{array}$ & VI \\
\hline
\end{tabular}




\begin{tabular}{|l|l|l|l|}
\hline & $\begin{array}{l}\text { vida e de vulnerabilidade dessa população, com o uso da modelagem } \\
\text { de equações estruturais. }\end{array}$ & corte transversal & \\
\hline 8 & $\begin{array}{l}\text { Analisar a relação da frequência de ocorrência do comportamento } \\
\text { simultâneo de temperaturas máximas diárias superiores a 30 }{ }^{\circ} \mathrm{C} \text { e } \\
\text { temperaturas mínimas superiores a } 20^{\circ} \mathrm{C} \text { e a vulnerabilidade social } \\
\text { como fatores de risco para mortalidade cardiovascular. }\end{array}$ & $\begin{array}{l}\text { Estudo } \\
\text { coorte }\end{array}$ & IV \\
\hline 9 & $\begin{array}{l}\text { Avaliar a associação dos fatores de risco para as doenças } \\
\text { cardiovasculares e qualidade de vida em universitários que } \\
\text { trabalham. }\end{array}$ & $\begin{array}{l}\text { Quantitativo, III } \\
\text { transversal e e } \\
\text { analítico }\end{array}$ & \\
\hline
\end{tabular}

Fonte: Elaborado pelos autores (2021).

A partir da leitura minuciosa dos resultados apresentados pelos artigos, foi identificada a relação entre as vulnerabilidades em saúde e as doenças cardiovasculares sendo as mais relatadas tabagismo, baixa escolaridade, consumo de bebidas alcoólicas, má alimentação, sedentarismo, obesidade, hipertensão arterial, diabetes mellitus, triglicerídeos altos e falta de acesso à informação (Quadro 3).

Quadro 3 - Caracterização das publicações incluídas na revisão, de acordo com o número e principais resultados. Sobral - CE, Brasil, 2021.

\begin{tabular}{|c|c|}
\hline $\mathbf{N}^{\mathbf{0}}$ & Principais Resultados \\
\hline 1 & $\begin{array}{l}\text { Alta prevalência de fumo nos participantes com baixa escolaridade, consumo de bebidas alcoólicas, má } \\
\text { alimentação, sedentarismo, obesidade, hipertensão arterial sistêmica, diabetes mellitus, nível de triglicerídeos } \\
\text { elevados, baixa escolaridade, não tinham acesso à informação ou condições de ter uma boa alimentação. }\end{array}$ \\
\hline 2 & $\begin{array}{l}\text { DCVs e uma tendência decrescente para os efeitos do frio. Na análise a magnitude crescente dos efeitos do calor } \\
\text { foi maior em homens do que mulheres e maiores em pessoas com idade } \leq 69 \text { anos do que naquelas com idade } \\
\geq 70 \text { anos. Destaca-se a necessidade urgente de preparações para ameaças emergentes à saúde cardiovascular de } \\
\text { altas temperaturas. }\end{array}$ \\
\hline 3 & $\begin{array}{l}\text { A exposição de curto prazo à Poluição do ar por partículas finas, mesmo em níveis abaixo dos limites } \\
\text { regulatórios atuais, pode estar associada ao aumento de internações hospitalares por DCV. Na China, a } \\
\text { exposição de curto prazo a partículas finas foi associada ao aumento de internações hospitalares para todas as } \\
\text { principais DCVs. }\end{array}$ \\
\hline 4 & $\begin{array}{l}10,9 \% \text { da amostra do estudo são pessoas que seguem uma trajetória de emprego formal, com alguns indivíduos } \\
\text { fazendo a transição entre empregos formais e informais ao longo da vida. } 100 \% \text { dos membros beberam e } \\
\text { fumaram regularmente durante longos períodos de idade adulta, passando a beber e fumar, em média, aos } 22 \text { e } \\
20 \text { anos, respectivamente, e foi a que já havia sofrido um ataque cardíaco ao longo da vida. Em sua maioria, } \\
\text { mulheres com ensino médico completo. }\end{array}$ \\
\hline 5 & $\begin{array}{l}\text { Os pacientes que apresentaram mais vulnerabilidade para o agravo de DCV foram: pacientes mais velhos e com } \\
\text { mais consultas ambulatoriais nos últimos } 12 \text { meses. Pacientes com mais hospitalizações nos últimos } 12 \text { meses } \\
\text { com sintomas depressivos e aqueles que eram afro-americanos. }\end{array}$ \\
\hline 6 & $\begin{array}{l}\text { Os bombeiros com alto risco cardiovascular evidenciaram associações significativamente mais fortes entre os } \\
\text { sintomas de sensibilidade à depressão e sintomas psiquiátricos, em relação àqueles com baixo risco } \\
\text { cardiovascular. }\end{array}$ \\
\hline 7 & $\begin{array}{l}\text { Há forte relação entre as condições de vida - composto pelos indicadores anos de estudo dos homens idosos, } \\
\text { percentual de idosos em domicílios com banheiro/água encanada e probabilidade de sobrevivência aos } 60 \text { anos - } \\
\text { com a taxa de mortalidade por doenças cardiovasculares dos homens idosos da região nordeste em } 2000 \text {. }\end{array}$ \\
\hline 8 & $\begin{array}{l}\text { A relação entre vulnerabilidade social-temperatura-mortalidade existe para a população colombiana avaliada } \\
\text { (com mais de } 60 \text { anos de idade). A alta temperatura está ligada ao índice de mortalidade por doenças } \\
\text { cardiovasculares. }\end{array}$ \\
\hline 9 & $\begin{array}{l}\text { Entre os universitários que trabalham o nível da qualidade de sono e o sedentarismo observados podem } \\
\text { comprometer a saúde e, consequentemente, a qualidade de vida dessa população, tornando-os mais vulneráveis } \\
\text { ao desenvolvimento de DCVs }\end{array}$ \\
\hline
\end{tabular}

Fonte: Elaborado pelos autores (2021). 


\section{DISCUSSÃO}

A partir da análise dos artigos percebeu-se que são múltiplos os fatores de vulnerabilidade que propiciam o adoecimento cardiovascular, dentre os quais destacam-se os fatores de risco que são bem divulgados e conhecidos pela população em geral: tabagismo, baixa escolaridade, consumo de bebidas alcoólicas, má alimentação, sedentarismo, obesidade, HAS, DM, triglicerídeos altos e falta de acesso à informação ${ }^{(16)}$. Isto se deve ao fato de que os maus hábitos de vida influenciam negativamente na qualidade de vida da população, assim a população que possui alguns desses maus hábitos de vida tornam-se mais vulneráveis ao desenvolvimento de DCV.

Nesse interim, a vulnerabilidade em saúde é uma situação de ameaça ao indivíduo, na qual antecede o risco na autonomia humana, colocando em situação frágil para o desenvolvimento de doenças, incapacidades, agravos, danos ou sofrimento, em contextos individuais, coletivos e contextuais ${ }^{(14)}$. Nessa perspectiva, estudos nos últimos anos, destacam que são inúmeras as definições do termo de vulnerabilidade, tendo conceito na educação, ciências econômicas e jurídica, psicologia e área da saúde, especificamente na saúde pública e coletiva. Em geral, o conceito de vulnerabilidade relata a condição humana e trajetória existencial das pessoas ${ }^{(17)}$.
No decorrer dos estudos nos últimos anos sobre vulnerabilidade em saúde, destacase a existência de três elementos que se relacionam para a caracterização da exposição ou susceptibilidade ao agravo, sendo elas: dimensão individual, social e programático. Logo, cada uma das dimensões é consentida como cenário no qual a vulnerabilidade se materializa $^{(18)}$.

Assim, o termo vulnerabilidade, destaca-se em diferentes campos disciplinares, sendo utilizado por órgãos internacionais e governamentais para a análise do processo saúde-doença. Dentro dos diversos campos de estudo, a vulnerabilidade trata-se de um estudo permanente para construção de conhecimento sobre ciências da vida, ciências naturais e sociais, em especial na área da saúde ${ }^{(19)}$.

Desta forma, a população em vulnerabilidade é a mais impactada pelos seus efeitos, sendo eles a ausência e/ou insuficiência de recursos e os problemas de saúde. Alguns estudos apontam, que a vulnerabilidade é uma condição de precariedade produzida pelas desiguais formas que o sujeito interagir no campo da saúde, sistema socioeconômico, demográfico, cultural, contextualização familiar e redes de suporte social de gênero, violência e controle social $^{(15)}$.

Ao realizar uma comparação entre a literatura utilizada para análise, pode-se afirmar que as populações de idosos, afro- 
americanos e das mulheres fumantes desde os 20-22 anos, são os mais susceptíveis para o agravamento cardiovascular $^{(20-22)}$. $\mathrm{O}$ tabagismo é ainda hoje um grande problema de saúde pública, visto que, causa danos muitas vezes irreparáveis ao sistema cardiorrespiratório, ademais, é importante ressaltar que boa parte da população que fuma, iniciou o uso do fumo cedo, isto, contribui para o desenvolvimento de DCV visto que, forma placas de gordura nos vasos sanguíneos, o que leva ao aumento da pressão arterial e a frequência cardíaca, e pode levar a formação de trombos ${ }^{(23)}$.

Sendo que, nos estudos de $\mathrm{Lu}$ et al. $(2020)^{(21)}$ e Benitez $(2020)^{(24)}$, afirma-se que a alta temperatura do ambiente e o envelhecimento, também são fortes fatores desencadeadores para as DCVs. Tal afirmativa complementa-se com o artigo de Wang et al. (2019) ${ }^{(20)}$, que aponta os idosos e o povo afro-americano, como mais vulneráveis a complicações cardiovasculares. Isto porque a população idosa possui um sistema imunológico mais frágil, tornando-os assim mais vulneráveis ao desenvolvimento de doenças. Aliado a isto, é importante considerar a história pregressa de cada pessoa.

No que se refere a temperatura ambiente, um estudo desenvolvido com base nos dados climatológicos de Ponta Grossa observou que as patologias do sistema cardiovascular são influenciadas tanto pelas altas como pelas baixas temperaturas, visto que, a temperatura do ar colabora para retirada do sistema cardiovascular de sua homeostasia e assim deixando-o propicio a ocorrência de patologias ${ }^{(25)}$. Além de alta temperatura, a poluição do ar pode ser citada como fator de risco para o desenvolvimento e agravo de doenças cardíacas ${ }^{(21,24,26)}$.

Dhungana et al. (2018) ${ }^{(16)}$, traz em seu estudo a baixa escolaridade como situação de vulnerabilidade, ainda que, pessoas com ensino médio completo também se encontram em circunstância de risco à saúde cardiovascular $^{(22)}$. No entanto, a situação socioeconômica é apontada como um fator vulnerabilizante, visto que, está muitas vezes aliado a falta de acesso à informação, bem como as más condições de vida que contribuem para o desenvolvimento de doenças, entre elas a DCV.

Nesse ínterim, pode-se vislumbrar que as situações de vulnerabilidade que causam o adoecimento cardiovascular envolvem fatores fisiológicos, sociais, econômicos e até mesmo ambientais. Dessa forma, despertando o olhar multidimensional para a busca de soluções que sanem e/ou minimizem tais circunstâncias de riscos para as DCVs.

Embora, afirme-se que a população idosa seja uma das mais suscetíveis, deve-se citar também que universitários que trabalham, e que possuem maus hábitos de vida são propensos ao adoecimento cardíaco $^{(27)}$. A má qualidade de vida trata-se de uma vulnerabilidade que pode ser 
caracterizada como uma das maiores causadoras de riscos cardíacos, pois tanto afeta os jovens como aos idosos ${ }^{(27,28)}$. Interessante comparar as diferentes faixas etárias afetadas, os idosos devido a fragilidade adquirida com o passar dos anos, e os jovens devido à sobrecarga de atividades, a exposição ao estresse constante que os torna vulneráveis da mesma forma que aos idosos.

Ranney et al. (2020) ${ }^{(29)}$, em seu estudo ao avaliar os bombeiros, revelou que os profissionais portadores de ansiedade $\mathrm{e}$ sensíveis a sintomas psiquiátricos, apresentam-se como um público com grande probabilidade de desenvolver doenças cardíacas. A partir da análise do estudo de Ranney et al. (2020) ${ }^{(29)}$, torna-se relevante observar a influência da rotina de trabalho na saúde física da população, bem como a relação com as doenças psiquiátricas, sendo importante ressaltar que corpo e mente estão sim interligados, portanto, deve-se olhar com mais atenção aos pacientes psiquiátricos, visto que, os sintomas ansiosos e depressivos podem levar ao desenvolvimento de $\mathrm{DCVs}^{(30)}$.

Logo, pode-se perceber que há uma consonância entre os estudos analisados, pois apresentam as situações de vulnerabilidades nos seus mais variados aspectos, complementando a importância de estudar sobre a temática para o desenvolvimento de medidas que viabilizem melhores estratégias para a prevenção e promoção da saúde cardiovascular ${ }^{(16,20-22,24,26-29)}$.

\section{CONSIDERAÇÕES FINAIS}

A partir do levantamento bibliográfico desenvolvido neste estudo, compreende-se que a vulnerabilidade em saúde proporciona a compreensão das condições da vida do indivíduo, logo, as DCV têm imposto desafios para o setor da saúde e para as políticas públicas envolvidas no combate aos seus fatores de risco, e estudos associados a vulnerabilidade em saúde.

Dentre os aspectos relacionados a vulnerabilidade em saúde no adoecimento cardiovascular destacam-se: tabagismo, baixa escolaridade, consumo de bebidas alcoólicas, má alimentação, sedentarismo, obesidade, hipertensão arterial, diabetes mellitus, triglicerídeos altos, falta de acesso à informação, exposição a alta temperatura e poluição do ar, histórico de internações hospitalares, depressão, sintomas psiquiátricos e qualidade do sono.

A principal limitação desta pesquisa esteve associada ao fato de que ainda existem poucos estudos sobre situação de vulnerabilidade em saúde em pessoas com doenças cardiovasculares. Nesse interim, sugerem-se integrar mais revisões da temática na literatura e investigações que envolvam atribuições para amenizar vulnerabilidade em saúde e prevenção a saúde cardiovascular. Portanto, com o presente estudo espera-se que a leitura desperte imersões científicas por 
outros pesquisadores para a compreensão e comprovação dos aspectos estudados.

\section{REFERÊNCIAS}

1. Lucas MG, Oliveira EBC, Oliveira IC, Basseto M, Machado RC. Impacto de uma capacitação para enfermeiros acerca da assistência no pós-operatório de cirurgia cardíaca. REV. SOBECC [internet]. abr./jun. 2018 [citado em 01 de junho de 2021]; 23 (2): 89-95. Disponível em: https://revista.sobecc.org.br/sobecc/art icle/view/381.

2. Santos AB, Perusulo EGR, Silva GJV; Oliveira GTS. O movimento sanitarista no Brasil. Caderno Humanidades em Perspectivas - II Simpósio de Pesquisa Social e II Encontro de Pesquisadores em Serviço Social - Edição Especial [internet]. Outubro de 2019 [citado em 02 de junho de 2021]; 6 (3): 307-321. Disponível em: https://www.cadernosuninter.com/inde x.php/humanidades/article/view/1211.

3. Florêncio RS, Moreira TMM. Modelo de vulnerabilidade em saúde: esclarecimento conceitual na perspectiva do sujeito-social. Acta Paul Enferm [internet]. 2021 [citado em 20 de maio de 2021]; 34: 1-15. Disponível em: https://www.scielo.br/j/ape/a/j5R4zLd BMPzwyPjKqYRHsFz/?format=pdf\& lang=pt.

4. Farias MS, Oliveira SC, Freitas MC, Guedes MVC, Silva LF, Barros LBF. Cuidado Clínico De Enfermagem No Cotidiano De Sua Prática E Em Saúde Cardiovascular. R. Enferm. UFJF [internet]. Jan./jun. de 2018 [citado em 19 de maio de 2021]; 4 (1): 77-82. Disponível em: https://periodicos.ufjf.br/index.php/enf ermagem/article/download/14019/751 8.
5. Frota KC, Ponte KMA, Souza FDC, Adriano CKS. Vulnerabilidades em saúde na reabilitação cardiovascular: relato de caso a partir da teoria de enfermagem. J. nurs. Health [internet]. 2020 [citado em 18 de maio de 2021]; 10 (2). Disponível em: https://periodicos.ufpel.edu.br/ojs2/ind ex.php/enfermagem/article/view/1801 4/11327.

6. Nichiatta LYI, Bertolozzi MR, Gryschek ALPL, et al. Potencialidade do conceito de vulnerabilidade para a compreensão das doenças transmissíveis. Rev Esc Enferm USP [internet]. 2011 [citado em 20 de maio de 2021]; 45(2):1769-73. Disponível em:

https://www.scielo.br/j/reeusp/a/w6Y v6Vg535yWtdDwWhyQ6gn/?lang=pt $\&$ format $=$ pdf.

7. Berardinelli LMM, Santos I, Santos MLCS, Lima TCL, Missio AC, Berardinelli LM. Identificando vulnerabilidade para complicações cardiovasculares em idosos: uma estratégia para cuidado. Rev. Enferm. UERJ [internet]. Out./dez. de 2011 [citado em 22 de maio de 2021];19 (4): 541-6. Disponível em: http://www.facenf.uerj.br/v19n4/v19n 4a06.pdf.

8. Malta DC, Teixeira R, Oliveira GMM, Ribeiro ALP. Mortalidade por Doenças Cardiovasculares Segundo o Sistema de Informação sobre Mortalidade e as Estimativas do Estudo Carga Global de Doenças no Brasil, 2000-2017. Arq. Bras. Cardiol. [internet]. Agosto de 2020 [citado em 01 de junho de 2021]; 115 (2). Disponível em: https://www.scielo.br/j/abc/a/mX3zD Ly43CbWt6sjm6J6GDc/abstract/?lang $=\mathrm{pt}$.

9. Cestari VRF. Vulnerabilidade da pessoa com Insuficiência Cardíaca: Elaboração de um instrumento de 
mensuração [dissertação na internet]. Fortaleza: Universidade Estadual do Ceará; 2017 [citado em 12 de junho de 2021]. 213p. Disponível em: http://www.uece.br/ppcclis/wpcontent/uploads/sites/55/2019/12/VIR NA.pdf.

10. Maniva SJCF, et al. Educational Technologies for health education on stroke: an integrative review. Rev Bras Enferm. [internet]. 2018 [citado em 08 de junho de 2021]; 71 (4): 1724-31. Disponível em: https://www.scielo.br/pdf/reben/v71s4 /pt_0034-7167-reben- 71-s4-1724.pdf.

11. Mendes KD, Silveira RCCP, Galvão CM. Uso de gerenciador de referências bibliográficas na seleção dos estudos primários em revisão integrativa. Texto contexto - enferm. [internet]. 2019 [citado em 10 de junho de 2021]; 28. Disponível em: http://www.scielo.br/scielo.php?script $=$ sci_arttext\&pid=S0104-

07072019000100602\&lng $=e n \& n r m=i$ so.

12. Santos CMC, Pimenta CAM, Nobre MRC. A estratégia pico para a construção da pergunta de pesquisa e busca de evidências. Rev Latinoamericana de Enfermagem [internet]. Mai./jun. 2007 [citado em 20 de junho de 2021]; 15 (3):1-4. Disponível em: http://www.scielo.br/pdf/rlae/v15n3/pt _v15n3a23.pdf.

13. Street B. Dimensões "escondidas" na escrita de artigos acadêmicos [internet]. 2010 [citado em 22 de novembro de 2021]; 28 (2): 541-67. Disponível em: https://periodicos.ufsc.br/index.php/pe rspectiva/article/view/2175-

795X.2010v28n2p541.

14. Ayres JRCM, et al. Risco, vulnerabilidade e práticas de prevenção e promoção da saúde. In: Campos GWS, et al. Tratado de Saúde Coletiva [internet]. $2^{\mathrm{a}}$ ed. HUCITEC.
2006 [citado em 25 de junho de 2021]. Disponível em: https://edisciplinas.usp.br/pluginfile.p $\mathrm{hp} / 347426 / \mathrm{mod}$ _resource/content/1/ris co_vulnearabilidade $\% 20$ Ayres $\% 20 \mathrm{e} \%$ 20cols.pdf.

15. Melnyk B, Fineoult-Overholt E. Evidence-based practice in nursing \& health care: a guide to best practice [internet]. Philadelphia: Lippincot Williams \& Wilkins, 2011 [citado em 20 de junho de 2021]. Disponível em: https://iucat.iu.edu/iuk/10205811.

16. Dhungana RR, et al. Prevalência de fatores de risco para doenças cardiovasculares: um estudo transversal baseado na comunidade em uma comunidade periurbana de Katmandu, Nepal. Indian Heart J. [internet]. Dezembro de 2018 [citado em 22 de junho de 2021]; 70 (3): 2027. Disponível em: https://www-ncbinlm-

nih.ez11.periodicos.capes.gov.br/pmc/ articles/PMC6309148/.

17. Cestari VRF, Moreira TMM, Pessoa VLMPP, Florêncio RS. Editora CRV. Insuficiência cardíaca interface com a vulnerabilidade em saúde [internet]. Curitiba, 2019 [citado em 01 de julho de 2021]. Disponível em: https://www.editoracrv.com.br/produt os/detalhes/34056-insuficienciacardiaca-brinterface-com-avulnerabilidade-em-saude.

18. Ayres JRCM. Vulnerabilidade, direitos humanos e cuidado: aportes conceituais. Editores: Barros S, Campos PFS, Fernandes JJS [internet]. Atenção à saúde de populações vulneráveis. Barueri, SP: Manole, 2014 [citado em 20 de setembro de 2021]. Disponível em: https://www.jurua.com.br/shop_item.a sp?id=22729.

19. Dimenstein M, Cirilo Neto MC. Abordagens conceituais da vulnerabilidade no âmbito da saúde e 
assistência social. Pesquisas e Práticas Psicossociais [internet]. 2020 [citado em 01 de outubro de 2021]; 15 (1). Disponível em: http://pepsic.bvsalud.org/pdf/ppp/v15n 1/02.pdf.

20. Wang J, Dietrich MS, Bell SP, Maxwell CA, Simmons SF, Kripalani S. Mudanças $\mathrm{Na}$ Vulnerabilidade Entre Pacientes Idosos Com Doença Cardiovascular Nos Primeiros 90 Dias Após A Alta Hospitalar: Uma Análise Secundária De Um Estudo De Coorte. BMJ Open [internet]. 2019 [citado em 07 de agosto de 2021]; 9 (1). Disponível em: https://www-ncbi-nlm-

nih.ez11.periodicos.capes.gov.br/pmc/ articles/PMC6352778/.

21. Lu P, Xia G, Zhao Q, Xu R, Li S, Guo $Y$. Temporal trends of the association between ambient temperature and hospitalisations for cardiovascular diseases in Queensland, Australia from 1995 to 2016: A time-stratified casecrossover study. PLoS Med. [internet]. 2020 [citado em 02 de julho de 2021]; 17 (7). Disponível em: https://www-ncbi-nlm-

nih.ez11.periodicos.capes.gov.br/pmc/ articles/PMC7373260/pdf/pmed.1003 176.pdf.

22. Madero-Cabib I, Azar A, Bambs C. Trajetórias de emprego ao longo da vida, uso de tabaco e consumo de álcool e doenças cardiovasculares na velhice. SSM Popul Health [internet]. Março de 2021 [citado em 10 de agosto de 2021]; 13. Disponível em: https://www-ncbi-nlm-

nih.ez11.periodicos.capes.gov.br/pmc/ articles/PMC7848642/.

23. Melo LD, Jeremias JS, Shubo AFMF, Taroco FE, Spindola T, Gomes Filho W, Ferreira BSB, Barbosa DJ. Smoking, Systemic Arterial Hypertension and Pandemic of COVID-19: A Freudian
Society and Development [internet]. 2020 [citado em 23 de agosto de 2021]; 9 (11). Disponível em: https://rsdjournal.org/index.php/rsd/art icle/view/10240.

24. Benítez DJR. Variabilidade da temperatura do ar diária, vulnerabilidade social e a relação com altas taxas de mortalidade por doenças cardiovasculares na Colômbia [tese na internet]. São Paulo: Universidade de São Paulo; 2020 [citado em 15 de julho de 2021]. 36 p. Disponível em: https://www.teses.usp.br/teses/disponi veis/6/6143/tde-23092020-

115034/publico/RoncancioBenitezDJ_ DR_R.pdf.

25. Galvão N, Leite ML, Virgens Filho JS, Pontes CC. Relação entre fatores climáticos e doenças do aparelho cardiovascular no município de ponta grossa-pr. Hygeia - Revista Brasileira de Geografia Médica e da Saúde [internet]. 28 de dezembro de 2015 [citado em 20 de julho de 2021]; 11 (21): 93 - 106. Disponível em: http://www.seer.ufu.br/index.php/hyge ia/article/view/30332.

26. Tian Y, et al. Associação entre poluição ambiente por partículas finas e internações hospitalares por causa de doença cardiovascular específica: estudo de série temporal em 184 grandes cidades chinesas. BMJ [internet]. 2019 [citado em 04 de julho de 2021]; 367: 365-72. Disponível em: https://www-ncbi-nlm-

nih.ez11.periodicos.capes.gov.br/pmc/ articles/PMC7190041/.

27. Vale MEG, Melo MLV, Isidório UA, Feitosa ANA, Sousa MNA, Araújo WA, Custódio PP, Assis EV. Fatores de risco cardiovasculares e qualidade de vida em universitários. Rev enferm UFPE on line [internet]. Outubro de 2018 [citado em 11 de agosto de 2021]; 12 (10) :2743-52. Disponível em:

Psychoanalytical Analysis. Research, 
https://periodicos.ufpe.br/revistas/revi staenfermagem/article/view/237491.

28. Santos JP, Paes NA. Associação entre condições de vida e vulnerabilidade com a mortalidade por doenças cardiovasculares de homens idosos do Nordeste. Rev. bras. epidemiol. [internet]. Junho de 2014 [citado em 08 de julho de 2021]; 17 (2). Disponível em: https://www.scielo.br/j/rbepid/a/qm36 6txMGdwCFz63Vy8x65m/?lang=pt\#.

29. Ranney RM, Bing-Canar H, Paltell KC, Tran JK, Berenz EC, Vujanovic AA. Risco cardiovascular como moderador de associações entre sensibilidade à ansiedade, tolerância ao estresse, PTSD e sintomas de depressão entre bombeiros expostos a traumas. Journal of Psychosomatic Research [internet]. Dezembro de 2020 [citado em 10 de agosto de 2021]; 139. Disponível em: https://wwwsciencedirect.ez11.periodicos.capes. gov.br/science/article/pii/S0022399 92030831X?via\%3Dihub.

30. Régis BN, Araújo RLR, Souza VG, Santiago Neto NA, Nodari NL, Hayasida NMA. Ansiedade, depressão e doença cardiovascular em jovens adultos: uma revisão da literatura. Revista Saúde e Desenvolvimento Humano [internet]. Maio de 2016 [citado em 20 de agosto de 2021]; 4 (1): 91-100. Disponível em: https://revistas.unilasalle.edu.br/index. php/saude_desenvolvimento/article/vi ew/2317-8582.16.22/pdf.

\section{Autor correspondente}

Ingrid Kelly Morais Oliveira, Av. Cmte. Maurocélio Rocha Pontes, 186 - Derby Clube, Sobral - CE, 62042-280, 88 99804-4173,

ingridkelly17.ik@gmail.com.

Submissão: 2021-11-08

Aprovado: 2021-11-23 